\section{Insect behaviour}

\section{Motion camouflage in dragonflies}

M ost animals can skilfully conceal themselves when stationary ${ }^{1}$, but they may become apparent as soon as they move. Here we use stereo cameras to reconstruct the movements in three dimensions of dragonflies (Hemianax papuensis), and show that these insects actively use motion camouflage to disguise themselves as stationary during territorial aerial manoeuvres. Deployment of this sophisticated technique by the oldest airborne predator tricks the victim's retina into perceiving the stalker as stationary even while it darts about in pursuit.

Optic flow - the apparent movement of objects as perceived using the retina - is a primary cue for detecting predators and prey. Predatory animals attacking stationary prey generally attempt to conceal their presence by approaching very slowly, sometimes relying on a camouflaging background. Active motion camouflage has been proposed as a strategy by which a predator can conceal its movements while shadowing or attacking highly manoeuvrable prey².

Motion camouflage can be achieved if one animal (the shadower) moves in such a way as to produce the same image motion on the retina of another animal (the shadowee) as would a stationary object in the environment. We reconstructed 15 threedimensional flight trajectories of interactions between conspecific dragonflies, of which six showed clear evidence of active motion camouflage.

Figure la shows an interaction between two male dragonflies: the lines connecting the heads of the shadower (blue dots) and shadowee (red dots) intersect in a small region behind the shadower. The centre of the shaded sphere represents the best estimate of the common point of intersection, and the radius of the sphere represents the uncertainty of this estimate.

If motion camouflage were perfect, all lines would intersect at a single point, and the sphere of uncertainty would have zero radius. In this example, the radius of the intersection sphere is $11.3 \mathrm{~mm}$. Given the \pm 10 - $\mathrm{mm}$ accuracy in measuring position at a filming distance of $1.5 \mathrm{~m}$, we may say that the lines intersect almost at a common point. This indicates that the shadower is emulating the trajectory of the projection of the fixed intersection point on the shadowee's retina. In other words, as far as the shadowee is concerned, the shadower is indistinguishable from a stationary object positioned at the intersection point.

The effectiveness of the strategy of motion camouflage is illustrated by a comparison of the apparent motion of the shadower on the

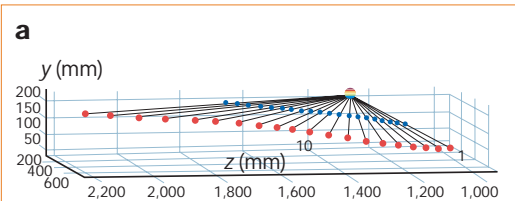

infinity - that is, the shadower imitates an object at infinity. This example reveals that a shadower can combine two different types of motion camouflage in a single episode.

Active motion camouflage requires the shadower to move in such a way that it imitates the trajectory of a fixed object on the retina of the shadowee by precise flight control and positional sensing ${ }^{4}$, although exactly how the shadower achieves this is unclear $^{2}$. Given the high sensitivity of the insect visual system to movement ${ }^{5}$, motion camouflage is likely to be the key to the success of such manoeuvres.

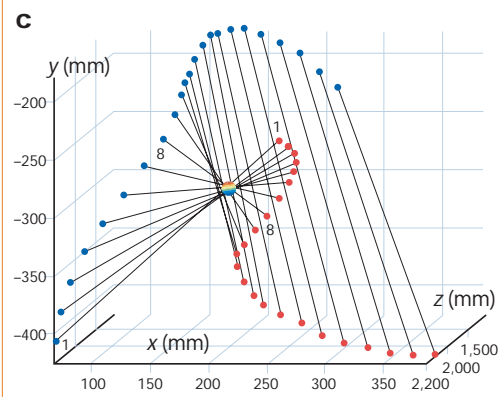

Akiko Mizutani*, Javaan S. Chahl*†, Mandyam V. Srinivasan*

*Centre for Visual Science, Research School of Biological Sciences, Australian National University, PO Box 475, Canberra, ACT 2601, Australia email: akiko@biorobotics.anu.edu.au †Weapons Systems Division, D efence Science and Technology Organisation, Edinburgh, South Australia 5111, Australia

1. Curio, E. The Ethology of Predation (Springer, Berlin, 1976). 2. Srinivasan, M. V. \& Davey, M. Proc. R. Soc. Lond. B 259, 19-25 (1995).

3. Collett, T. S. \& Land, M. F. J. Comp. Physiol. 99, 1-66 (1975) 4. US Office of Naval Research Insect Vision Based Seekers and Controllers for Guided Weapons (contract N 68936-1-2-001)

Figure 1 Examples of active motion camouflage. a, Threedimensional reconstruction of a territorial interaction involving two male dragonflies. The reconstruction was achieved by using calibrated $^{6}$ and synchronized (to within $10 \mu \mathrm{s}$ ) stereo cameras. Positions of the heads of the shadower (blue dots) and shadowee (red dots) are connected by lines at corresponding 20-ms steps. The connecting lines converge behind the shadower; the point of convergence indicates the apparent position of the shadower to the shadowee. The shaded sphere (radius, $11.3 \mathrm{~mm}$ ) represents the uncertainty of the 'best' estimate of the intersection point. b, Angular-velocity profile produced by the shadowing dragonfly in the shadowee's eye (filled circles) compared with that produced by a virtual stationary object at the intersection point (hollow circles). c, Another example of motion camouflage. The connecting lines converge in front of the shadower during frames 1-8, after which the shadower begins to imitate an infinitely distant object. The radius of the intersection sphere is $8.5 \mathrm{~mm}$.

shadowee's retina with that produced by a virtual, stationary object positioned at the estimated intersection point (Fig. 1b). The two angular-velocity profiles are very similar, indicating that the strategy is likely to be effective in concealing the motion of the shadower.

In other male-male interactions, we observed variations in motion camouflage (Fig. 1c). During the first eight frames of filming, the lines connecting the two insects intersect at a small region between them; the shadower is therefore emulating a fixed object in front of it. Here the shadowing animal is not flying towards the shadowee, but is moving in the opposite direction. This is evidence that the flight pattern of the shadower is motivated by motion camouflage and is not an artefact or by-product of chasing per $\mathrm{se}^{2,3}$. After frame nine, the intersection point shifts to
5. Srinivasan, M. V. in Visual M otion and its Role in the Stabilization of Gaze (eds Miles, F. A. \& Wallman, J.) 139-155 (Elsevier, Amsterdam,1993).

6. Tsai, R. Y. IEEE J. Robot. Automat. 3, 323-344 (1987). Competing financial interests: declared none.

\section{Electromagnetic waves}

\section{Negative refraction by photonic crystals}

1 aterials that can bend light in the opposite direction to normal ('lefthanded' materials) reverse the way in which refraction usually works this negative refractive index is due to simultaneously negative permeability and permittivity $^{1-3}$. Here we demonstrate negative refraction of electromagnetic waves in a two-dimensional dielectric photonic crystal that has a periodically modulated unity $^{4-6}$. This experimental verification of negative refraction is a step towards the realization of a 'superlens' that will be able to focus features smaller than the wavelength of light.

Our structure consists of a square array of alumina rods in air; the calculated band diagram for this structure is shown in Fig. 1a. To obtain negative refraction, equalfrequency surfaces are needed for the photonic crystal that are both convex and larger than those for air (Fig. 1b). Note that conservation of the surface-parallel wave vector gives the direction of the refracted waves inside the photonic crystal (Fig. 1b).

We made transmission measurements positive permittivity and a permeability of 

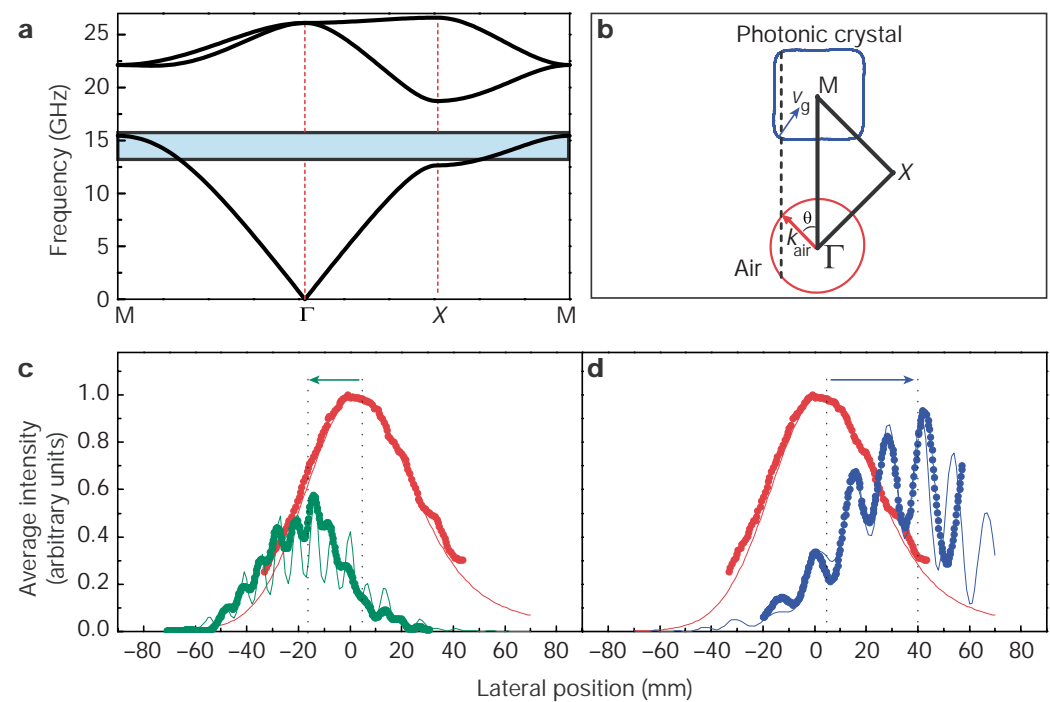

Figure 1 Comparison of positive and negative refraction. a, Band diagram for our structure for transverse magnetic polarization. Shading denotes the frequency range in which negative refraction occurs. b. Equal-frequency contours in $k$ space of air and of the photonic crystal at $13.7 \mathrm{GHz} . \theta$ is the angle of incidence from the air to the crystal; $v_{g}$ is the group velocity inside the photonic crystal. c, Negative refraction. Average intensities were calculated at the second interface with the photonic crystal (green) and at the first interface without the photonic crystal (red), and corresponding power distributions were measured. d, Positive refraction. Average intensities were calculated at the second interface with a slab containing polystyrene pellets (blue) and at the first interface without the slab (red), and the corresponding power distributions were measured. Arrows in $\mathbf{c}$ and $\mathbf{d}$ indicate the refracted beam's direction of divergence from the incident beam.

to confirm our structure's predicted negative refraction, using the interfaces of the photonic crystal in the $\Gamma-M$ direction. The electric field was kept parallel to the rods for all measurements and calculations; the horn antenna was orientated so that the incident waves make an angle of $45^{\circ}$ with the normal of the $\Gamma-M$ interface. Our structure exhibits the maximum angular range of negative refraction at an operating frequency of 13.7 gigahertz (GHz). In simulations using the finite-difference time-domain method (FDTD), the incident gaussian beam width was set at $6 \mathrm{~cm}$, which is equal to the width of the horn antenna.

The centre of the outgoing gaussian beam is shifted to the left of the centre of the incident gaussian beam (Fig. 1c), which corresponds to negative refraction ${ }^{7}$. The negative index of refraction was determined to be -1.94 , which is close to the theoretical value of -2.06 calculated by the FDTD method. For comparison, we repeated the measurements and simulations with a slab containing only polystyrene pellets, which has a refractive index of 1.46 , and found the refracted beam to be on the right of the incident beam, corresponding to a positive index of 1.52 (Fig. 1d).

The advantage of negative refraction in the valence band is that there is no Bragg reflection; such reflections occur in higher bands of the photonic crystal, and we have a well-defined, single-beam propagation that is negatively refracted. Another advantage of operating in the valence band is that the $63 \%$, which is almost three orders of magnitude larger than the typical transmission efficiency in a left-handed material ${ }^{2,3}$. The negative refraction effect that we describe depends only on the refractive index of the dielectric material and on the geometric factors used in two-dimensional photonic crystals. This effect can therefore also be observed at optical wavelengths, at which it is possible to obtain similar refractive indices by using transparent semiconductors.

Ertugrul Cubukcu*, Koray Aydin*, Ekmel Ozbay*, Stavroula Foteinopoulout, Costas M. Soukoulist

*D epartment of Physics, Bilkent University, Bilkent, 06533 Ankara, Turkey e-mail: cubukcu@fen.bilkent.edu.tr †Ames Laboratory, US D epartment of Energy, and Department of Physics and Astronomy, Iowa State University, Ames, lowa 50011, USA $\ddagger$ Research Center of Crete and Department of $M$ aterials Science and Technology, University of Crete, Heraklion, 71100 Crete, Greece

1. Pendry, J. B., Holden, A. J., Robins, D. J. \& Stewart, W. J. IEEE Trans. M icrowave Theory Tech. 47, 2075-2084 (1999).

2. Shelby, R. A., Smith, D. R. \& Schultz, S. Science 292, 77-79 (2001)

3. Bayindir, M., Aydin, K., Ozbay, E., M arkos, P. \& Soukoulis, C. M. Appl. Phys. Lett. 81, 120-122 (2002).

5. Luo, C., Johnson, S. G., Joannopoulos, J. D. \& Pendry, J. B. Phys. Rev. B 65, 201104(R) (2002).

6. Foteinopoulou, S., Economou, E. N.\& Soukoulis, C. M. Phys Rev. Lett. 90, 107402 (2003).

7. Kong, J. A., Wu, B. I. \& Zhang, Y. Appl. Phys. Lett. 80, 2084-2086 (2002).

Competing financial interests: declared none. transmission efficiency at this frequency is

4. Notomi, M. Phys. Rev. B 62, 10696-10705 (2000).
COMMUNICATIONS ARISING

Social networks

\section{Sexual contacts and epidemic thresholds}

P stributions of the number of sexual partners reported in surveys show a pronounced skew, with most people having had one or no partners in the past year and a small fraction having had many ${ }^{1,2}$. Liljeros and colleagues ${ }^{3}$ infer from the results of a Swedish survey that there is a "scale free" population distribution of sexual contacts, consistent with a preferentialattachment model ${ }^{3,4}$, in which "the rich get richer" and epidemics are driven by extremely promiscuous individuals. Here we reanalyse the data from Sweden and from other countries, using more appropriate statistical tools. Our findings support the conventional wisdom that epidemic thresholds exist in these populations, and indicate that current public-health strategies to reduce the spread of HIV and other sexually transmitted infections do not need to be radically refocused.

An important epidemiological question for highly skewed partnership distributions is whether their variance is finite $e^{5,6}$. As the reproduction number of a pathogen increases linearly with the variance in the level of sexual activity ${ }^{7,8}$, populations with infinite variance lack epidemic thresholds. In these populations, a sexually transmitted infection could persist regardless of its transmissibility, and interventions such as vaccines or barrier contraceptives would be ineffective for eradicating it.

Liljeros et al. estimate the scaling exponent for Sweden by fitting a line to the apparently linear region of the upper tail of the logged sexual-contact distribution. This approach is not statistically appropriate, for several reasons ${ }^{9}$. Inference on the basis of the distribution's extreme upper tail yields wildly increasing confidence intervals because there is little empirical information in this region (Fig. 1). Estimates based on partial lifetime contacts (as in Fig. 2b of ref. 3) are a source of further difficulties, including temporal confounding and censoring.

We take a more principled statistical approach, using a stochastic mechanism for the underlying preferential-attachment process. This yields a Yule distribution ${ }^{10}$, and infinite variance when the single scaling parameter $\rho \leqslant 3$. (Details of the Yule model and the statistical estimation procedure are presented elsewhere ${ }^{9}$.) We generalize the simple Yule model to allow for a mixture of distributions - a process for the lower tail, and a preferentialattachment-type process for the upper tail. Assuming the Yule form, we can estimate the model parameter using maximum 